\title{
Asymmetric Landau bands due to spin-orbit coupling
}

\author{
Sigurdur I. Erlingsson ${ }^{1}$, Andrei Manolescu ${ }^{1}$, and D. C. \\ Marinescu ${ }^{2}$ \\ ${ }^{1}$ School of Science and Engineering, Reykjavik University, Menntavegur 1, IS-101 \\ Reykjavik, Iceland \\ 2 Department of Physics and Astronomy, Clemson University,Clemson, South \\ Carolina 29634, USA
}

\begin{abstract}
We show that the Landau bands obtained in a two-dimensional lateral semiconductor superlattice with spin-orbit coupling (SOC) of the Rashba/Dresselhaus type, linear in the electron momentum, placed in a tilted magnetic field, do not follow the symmetry of the spatial modulation. Moreover, this phenomenology is found to depend on the relative tilt of magnetic field and on the SOC type: a) when only Rashba SOC exists and the magnetic field is tilted in the direction of the superlattice b) Dresselhaus SOC exists and the magnetic field is tilted in the direction perpendicular to the superlattice. Consequently, measurable properties of the modulated system become anisotropic in a tilted magnetic field when the field is conically rotated around the $z$ axis, at a fixed polar angle, as we demonstrate by calculating the resistivity and the magnetization.
\end{abstract}

PACS numbers: 71.70.Di, 71.70.Ej, 73.21.Cd 


\section{Introduction}

For more than ten years, the complex phenomenology observed in two dimensional semiconductor structures with spin-orbit coupling (SOC) has been at the forefront of numerous experimental and theoretical explorations [1]. Originating in the asymmetry of the quantum well or in the inversion asymmetry of the crystal structure, the spin orbit interaction, of the Rashba [2] and Dresselhaus [3] type, respectively, describes the coupling of the electron spin to its momentum, generating the possibility of simultaneous spin and charge effects, as well as the possibility of controlling the spin through the application of electric fields [4, 5]. It is the latter reason that underlies the continuous interest, because of a potential realization of semiconductor based spintronic devices. From this perspective, the investigation of magnetotransport properties was focused from the very beginning on identifying the best set-up which, in the presence of electric or magnetic fields, can showcase spin-dependent macroscopic response functions, and in particular spin or spin-polarized currents.

The transport theory of Landau levels in the presence of electrostatic modulation goes already back a quarter of a century [6, 7]. Since then these systems have attracted continued attention. In the more recent years of spintronics the effects of SOC were added [8], modulation effects on the spin Hall conductance were studied [9], and the effects of a tilted magnetic field were considered [10]. As has been pointed out, the spinorbit effect can be relatively enhanced in a tilted magnetic field, as observed both in transport [11] and magnetization measurements [12, 13]. The origin of this enhancement is that at Landau level crossing, where the orbital and Zeeman energies are equal, the Landau levels are linear with the SOC strength, and the spin mixing is maximized [12]. The suppression of orbital energy can also be achieved in one dimensional systems with periodic modulation where it has been proposed that signature of SOC can be measured in two terminal transport [14].

The experimental detection of the SOC in a nanostructure is in general difficult. The response functions depend on the energy dispersion, which usually include some weak effects of the SOC. An interesting situation was described in Ref. [15] where the energy spectrum of a two-dimensional (2D), laterally symmetric quantum wire, was calculated. Due to the interplay of SOC and an in-plane magnetic field perpendicular to the length of a wire the energy dispersion becomes asymmetric with respect to the sign reversal of the momentum along the wire [16]. The authors of Ref. [15] predicted that this situation can be experimentally verified by phototransport measurements, where an asymmetric band structure is essential for obtaining photocurrents [17]. Nevertheless, to our knowledge such experiments are not reported for quantum wires or another nanostructure with lateral confinement, where the electrons are the current carriers. Photoconductivity had been studied in the two-dimensional electron gas with SOC, but based on a different mechanism, of an imbalanced population of electrons with different spin orientation under the action of light and an in-plane magnetic field [18]. Only in a recent study magnetophotocurrents with possible spin polarization have been 
generated by electron-hole excitations in asymmetric Rashba spin-split bands in layered semiconductor BiTeI [19]. More complicated asymmetries of the energy spectra, for example due to the simultaneous presence of Rashba and Dresselhaus SOC in thick quantum wells in magnetic field, can generate equilibrium spin currents which are still not observed in experiments [20].

In the present paper we propose alternative possibilities to detect the effect on the energy dispersion resulting from the combination of SOC and an in-plane magnetic field in a $2 \mathrm{D}$ electron system (2DES). We consider a tilted magnetic field with a vertical component that is strong enough to induce the quantization of the Landau levels in the electron plane. We also consider a lateral modulation in the plane obtained by applying a periodic electrostatic potential along one direction in the plane, as shown in Fig. 1. We show that in the presence of SOC the resulting Landau bands, and implicitly the density of states, depend on the orientation of the in-plane magnetic field relatively to the direction of the modulation. In particular, if the electrostatic modulation has an inversion symmetry, the Landau bands do not obey it. We calculate the conductivity tensor and the total energy of the modulated 2DES and show that the presence of the SOC could be observed in magnetotransport and magnetization experiments, in a tilted magnetic field rotating conically around the normal direction to the plane.

\section{The weakly modulated $2 \mathrm{DES}$ in a tilted field}

In the following discussion we consider the 2DES situated in the $\hat{x}-\hat{y}$ plane, and subjected to a lateral modulation along the $\hat{x}$ direction which is induced by applying a periodic potential, $V(x)=V_{0} \cos Q x$. A magnetic field of magnitude $B$, tilted in respect with the $\hat{z}$ axis is also applied. Its direction is described by the usual angular representation, $\boldsymbol{B}=B(\cos \varphi \sin \theta, \sin \varphi \sin \theta, \cos \theta)$, as shown in Fig. 1. We assume that the perpendicular component of the magnetic field is strong enough such that the quantization of the single particle energies into Landau levels is induced. With $a$ and $a^{\dagger}$ the usual destruction and creation operators of the harmonic oscillator, the single particle Hamiltonian is written in a spin basis provided by the Pauli matrices, $\left\{\sigma_{x}, \sigma_{y}, \sigma_{z}\right\}$, as,

$$
H_{2 \mathrm{D}}=\hbar \omega_{c}\left(a^{\dagger} a+1 / 2\right)+\frac{g \mu B}{2} \boldsymbol{B} \cdot \boldsymbol{\sigma}+V(x+\xi),
$$

where $\xi=-\ell_{c}^{2} p_{y} / \hbar$ is the so called center coordinate, $\ell_{c}=\sqrt{\hbar / e B}$ is the magnetic length, and a unitary transformation $e^{i p_{x} \xi / \hbar}$ was used to displace the origin of the modulation potential. In the absence of SOC the orbital and spin states decouple, the spin states being either parallel or anti-parallel with the total magnetic field,

$$
\begin{aligned}
& \left|\uparrow_{\boldsymbol{B}}\right\rangle=\cos \frac{\theta}{2} e^{i \frac{\varphi}{2}}|\uparrow\rangle+\sin \frac{\theta}{2} e^{-i \frac{\varphi}{2}}|\downarrow\rangle, \\
& \left|\downarrow_{\boldsymbol{B}}\right\rangle=-\sin \frac{\theta}{2} e^{i \frac{\varphi}{2}}|\uparrow\rangle+\cos \frac{\theta}{2} e^{i \frac{\varphi}{2}}|\downarrow\rangle,
\end{aligned}
$$


where $|\uparrow\rangle$ and $|\downarrow\rangle$ are the usual eigenstates of $\sigma_{z}$. For a modulation amplitude much smaller than the cyclotron energy, i. e. $V_{0} \ll \hbar \omega_{c}$, the orbital states are obtained in first order perturbation theory to be,

$$
\begin{aligned}
|n, \xi\rangle & \approx|n\rangle-\frac{V_{0} \sin Q \xi}{\hbar \omega_{c}}\left(S_{n, n-1}|n-1\rangle-S_{n, n+1}|n+1\rangle\right) \\
& \left.-\frac{V_{0} \cos Q \xi}{2 \hbar \omega_{c}} C_{n, n-2}|n-2\rangle-C_{n, n+2}|n+2\rangle\right),
\end{aligned}
$$

where $S_{n, m}=\langle n|\sin Q x| m\rangle$ and $C_{n, m}=\langle n|\cos Q x| m\rangle$ are matrix elements in the basis of the usual harmonics oscillator states $|n\rangle$. By increasing the tilt of the magnetic field, such that the relative contribution of the in-plane component to the Zeeman splitting is enhanced, opposite-spin Landau bands cross [21].

In the presence of the Rashba interaction, of strength $\alpha$, the single-particle Hamiltonian acquires an additional term which reflects the coupling between the spin $\sigma$ and the electron momentum written in terms of the harmonic oscillator operators,

$$
H_{\mathrm{R}}=\frac{\alpha}{\sqrt{2} \ell}\left(a \sigma_{+}+a^{\dagger} \sigma_{-}\right)
$$

where $\sigma_{ \pm}=\sigma_{x} \pm i \sigma_{y}$. In these conditions the particle spin is not a good quantum number and the energy bands couple in the spin space. They can be determined by projecting the full Hamiltonian $H_{2 \mathrm{D}}+H_{\mathrm{R}}$ onto the subspace $|n, \xi\rangle\left|\uparrow_{\boldsymbol{B}}\right\rangle$ and $|n-1, \xi\rangle\left|\downarrow_{\boldsymbol{B}}\right\rangle$, followed by the diagonalization of the resulting $2 \times 2$ matrix. $H_{2 D}$ is trivially diagonal, with diagonal elements

$$
\begin{aligned}
& \varepsilon_{n, \uparrow_{B}}^{0}=\hbar \omega_{c}(n+1 / 2)-\frac{g \mu B}{2}+V_{n} \cos Q \xi \\
& \varepsilon_{n-1, \downarrow_{B}}^{0}=\hbar \omega_{c}(n-1 / 2)+\frac{g \mu B}{2}+V_{n-1} \cos Q \xi
\end{aligned}
$$

which are the standard Landau bands in the first order of the modulation strength, where $V_{n}=V_{0} C_{n, n}$.

The Rashba term generates

$$
\begin{aligned}
\frac{H_{R, n}}{\hbar \omega_{c}} & =\frac{\alpha n \cos ^{2} \frac{\theta}{2}}{\sqrt{2} \ell_{c}}\left(e^{i \varphi} \sigma_{+}+\text {h.c. }\right)+\frac{\alpha}{\sqrt{2} \ell_{c}} \frac{V_{0}}{\hbar \omega_{c}} \frac{\mathcal{S}_{n}+\mathcal{S}_{n-1}}{2} \\
& +\frac{\alpha}{\sqrt{2} \ell_{c}} \frac{V_{0}}{\hbar \omega_{c}}\left(\mathcal{O}_{z, n} \sigma_{z}+\mathcal{O}_{-, n} \sigma_{+}+\mathcal{O}_{-, n}^{*} \sigma_{-}\right),
\end{aligned}
$$

where $\mathcal{S}_{n}=\sqrt{n} S_{n, n-1}-\sqrt{n+1} S_{n, n+1}$ and

$$
\begin{aligned}
& \mathcal{O}_{z, n}=-\frac{\mathcal{S}_{n}-\mathcal{S}_{n-1}}{2} \sin \theta \cos \varphi \sin Q \xi, \\
& \mathcal{O}_{-, n}=\frac{\sqrt{n-1} C_{n, n-2}}{2} \sin ^{2} \frac{\theta}{2} e^{-i \varphi} \cos Q \xi .
\end{aligned}
$$

In the absence of the periodic potential $\left(V_{0}=0\right)$ the Rashba contribution reduces to the first term of Eq. (6) which, for a perpendicular magnetic field $(\theta=0)$ leads to the known exact eigenstates, since the Rashba interaction only couples two adjacent Landau levels [22]. As long as we are working in the regime $\hbar \omega_{c} \gg V_{0} \gg \frac{\alpha}{\sqrt{2} \ell}$ the Landau bands due 


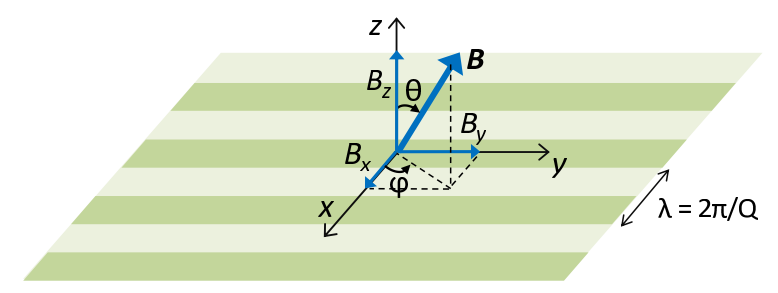

Figure 1. (Color online) A 2DES situated in the $x-y$ plane exhibits a density modulation (stripes) when subjected to a periodic potential $V_{0} \cos Q x$. The magnetic field, represented by the blue thick arrows, is oriented along a direction determined by the polar and azimuthal angles, $\theta$ and $\varphi$, respectively.

the effective modulation are obtained by diagonalizing each $2 \times 2$ subspace for Landau level $n$ [23]. The other terms of Eq. (6) are generated by the periodic potential. The second term is simply an energy shift which depends on the Landau level (via $\mathcal{S}_{n}$ ). The terms containing $\mathcal{O}_{z, n}$ and $\mathcal{O}_{-, n}$ can be interpreted as, respectively, the perpendicular and in-plane components of an effective, Landau level dependent magnetic modulation that appears due to the interplay of the Rashba SOI and the modulation potential. It is clear from Eq. (7) that the perpendicular component of the effective magnetic modulation leads to Landau bands that are asymmetric around $Q \xi=0$ or $Q \xi=\pi$, on account of the $\sin Q \xi$ term, although the modulation potential is an even function of $x$. Symmetric bands, i. e. even functions of $\xi$, are recovered only in the case where $\cos \varphi=0$, i. e. for $\varphi=\pi / 2$.

In the case of Dresselhaus type of SOC, of strength $\beta$, which can be written in a form equivalent to the Rashba coupling by using the spin rotation $e^{i \frac{\pi}{2} \sigma_{x}} e^{i \frac{\pi}{4} \sigma_{z}}$, one obtains very similar results for the Landau bands. Due to this well know Rashba-Dresselhaus transformation, the Landau bands obtained with $\alpha, \beta$, and $\left(B_{x}, B_{y}, B_{z}\right)$ are identical to those obtained with $\beta, \alpha$, and $\left(B_{y}, B_{x},-B_{z}\right)$.

To conclude this section we notice that, according to Eqs. (7)-(8), the amplitude of the Landau bands depends on the strength of the SOC and on the orientation of the in-plane magnetic field relatively to the modulation direction. In particular, if the external potential is an even function of $x$, the expected even parity of the Landau bands in the Brillouin zone is broken by the Rashba SOC if $B_{x} \neq 0$, or by the Dresselhaus $\mathrm{SOC}$ if if $B_{y} \neq 0$.

\section{The strongly modulated 2DES}

In order to increase the effect of the SOC on the energy dispersion we consider a modulation potential with an amplitude much larger that the cyclotron energy which can generate higher harmonics in the dispersion of the energy bands. To be more realistic we also include in the band structure calculations the Coulomb interaction between the electrons. We use the Hartree-Fock approximation where the Fock exchange is screened 

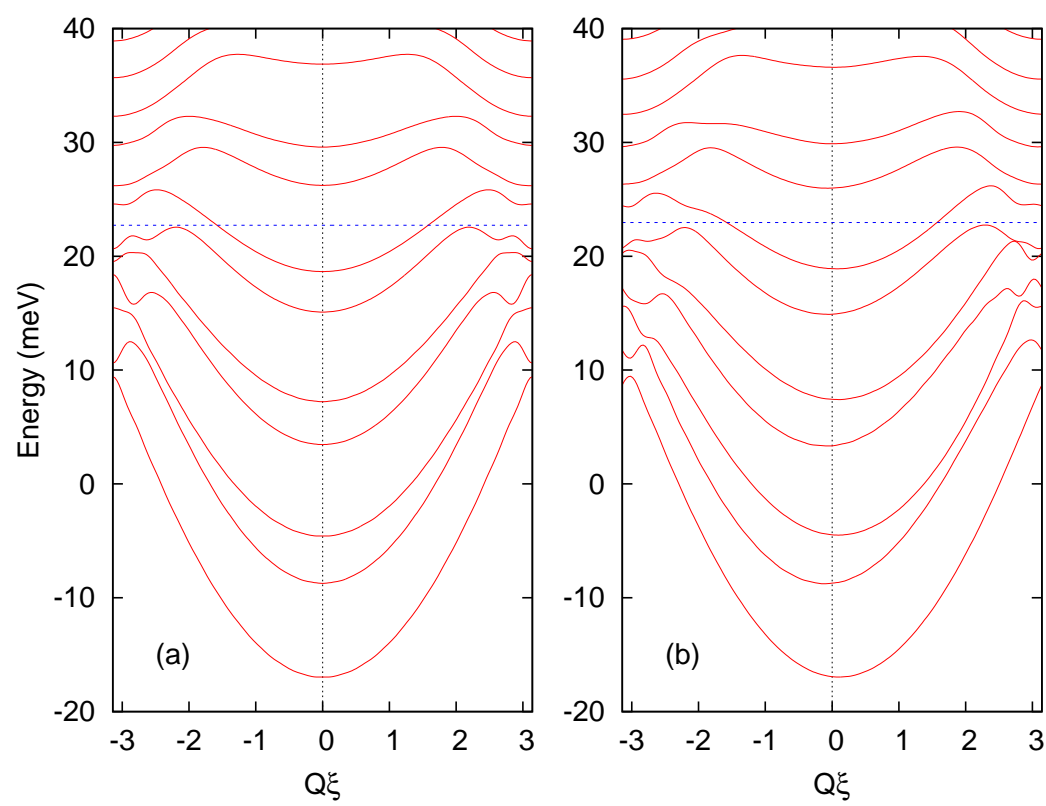

Figure 2. (Color online) Landau bands created by a modulation potential along the $x$ direction, with period $\lambda=150 \mathrm{~nm}$ and amplitude $V_{0}=40 \mathrm{meV}$, vs. the center coordinate $\xi$. The total magnetic field is $B=9.02 \mathrm{~T}$ and the polar angle $\theta=80.2^{\circ}$.

(a) The magnetic field is tilted in the $y$ direction, i. e. $B_{x}=0$, or $\varphi=\pi / 2$, and the Landau bands are symmetric within the Brillouin zone $-\pi<Q \xi<\pi$, where $Q=2 \pi / \lambda$. (b) The magnetic field is tilted in the $x$ direction, i. e. $B_{y}=0$, or $\varphi=0$, and the Landau bands become asymmetric. This asymmetry is due to the Rashba SOC. The horizontal dashed line indicates the Fermi energy. The material parameters correspond to InAs, with SOC $\alpha=20 \mathrm{meV} \mathrm{nm}$ and $\beta=0$

with the static polarizability. The long-range direct Coulomb term tends to screen the external potential and reduce the energy dispersion of the Landau bands, whereas the short-range exchange term tends to increase the dispersion by contributing with negative energy to the occupied states [24].

Examples of resulting Landau bands are shown in Fig. 2, for a modulation potential of period $\lambda=150 \mathrm{~nm}$ and amplitude $V_{0}=40 \mathrm{meV}$. The material parameters are chosen as for InAs: effective mass $m_{\text {eff }}=0.023 m_{0}$, effective g-factor $g_{\text {eff }}=-14.9$, Rashba SOC strength $\alpha=20 \mathrm{meV} \mathrm{nm}$. The density of electrons is $2.4 \times 10^{11} \mathrm{~cm}^{-2}$. In order to maximize the effect of the SOC we tilt the magnetic field such that the Zeeman and cyclotron energies are equal in the absence of the modulation, which allows the strongest mixing of spin up and spin down states. The corresponding polar angle is $\theta=80.2^{\circ}$. The total magnetic field is $B=9.02 \mathrm{~T}$. The Brillouin zone corresponds to center coordinate interval $(-\pi / Q, \pi / Q)$. As expected from the perturbative limit, if the magnetic field is tilted in the $y$ direction only, the bands are symmetric relatively to the center of the Brillouin zone, i. e. they are even functions of $\xi$. Whereas if the magnetic field is tilted along $x$ the bands become asymmetric, due to the Rashba SOC.

Consequently observables that depend on the energy structure should be anisotropic relatively to a rotation of the magnetic field around the $z$ axis, i. e. with a fixed polar 
angle $\theta$. In the following we describe such results for the electrical conductivity and for the magnetization of the modulated 2DES endowed with SOC.

\section{Conductivity}

We performed the calculations of the conductivity tensor, $\sigma_{x x}, \sigma_{y y}, \sigma_{x y}$, using the Kubo linear response formula. The longitudinal conductivity along the modulation, $\sigma_{x x}$, depends on the collision-induced broadening of the Landau bands which is taken into account within the self-consistent Born approximation, assuming delta-scatterers [7]. Following the result for the uniform 2DES we assume a broadening $\Gamma=\gamma \sqrt{B_{z}}$, with $\gamma=0.15 \mathrm{meV} / \mathrm{T}^{1 / 2}$. The longitudinal conductivity parallel to the modulation strips, $\sigma_{y y}$, is dominated by the drift of the electrons along the strips, which is known as band conductivity, but also incorporates a weaker scattering component [7]. The scattering conductivity is (approximately) proportional to the density of states at the Fermi level squared and therefore its maxima correspond to the van Hove singularities of the energy bands. In contrast, the band conductivity is inversely proportional to the same quantity, and therefore has maxima in the middle of the Landau bands. In a similar manner the scattering conductivity is proportional to $\Gamma^{2}$ whereas the band conductivity is proportional to $\Gamma^{-2}$ [24].

We treat the Hall conductivity in the simplest possible way, with a filling-factor $(\nu)$ dependent formula, $\sigma_{x y}=\left(e^{2} / h\right) \nu$, which does not describe Hall plateaus, but it is still a qualitatively reasonable result for a strong modulation. Since usually in transport experiments the resistivities are measured, rather than conductivities, we display in Fig. 3 the longitudinal resistivities

$$
\rho_{x x, y y}=\frac{\sigma_{y y, x x}}{\sigma_{x x} \sigma_{y y}+\sigma_{x y}^{2}} .
$$

In general $\sigma_{x x} \sigma_{y y} \ll \sigma_{x y}^{2}$ [7, 24] and therefore $\rho_{x x} \sim \sigma_{y y}$ and $\rho_{y y} \sim \sigma_{x x}$. On account of the opposite relation between conductivities and density of states the two resistivities oscillate with the magnetic field in antiphase, as can be seen by comparing Figs. 3 a-b or c-d, respectively. For example, in Fig. 2 the $z$ component of the magnetic field is $B_{z}=B \cos \theta=1.54 \mathrm{~T}$. Correspondingly, in Fig. 3 we notice a succession of a local maximum and a local minimum for $1.50 \mathrm{~T}<B_{z}<1.55 \mathrm{~T}$ for $\rho_{x x}$ (which is dominated by the band conductivity via $\sigma_{y y}$ ), since the density of states at the Fermi level increases by approaching the top of a Landau band, and the other order, first a minimum and then a maximum for $\rho_{y y}$ (which is dominated by the scattering conductivity $\sigma_{x x}$ ). The large dispersion of the Landau bands and the relatively small disorder broadening determine a value for $\rho_{y y}$ that is three orders of magnitude smaller than $\rho_{x x}$.

In the transport calculations we included a Dresselhaus SOC with $\beta=3 \mathrm{meV} \mathrm{nm}$, but that has no significant contribution in Fig. 3, More important, by increasing the Rashba SOC strength, from $\alpha=20$ to $\alpha=30 \mathrm{meV} \mathrm{nm}$, the resistivities change quite a lot. Both dispersion and asymmetry of the Landau bands increase with increasing SOC. The behavior of the resistivities is complex and rather difficult to interpret. 

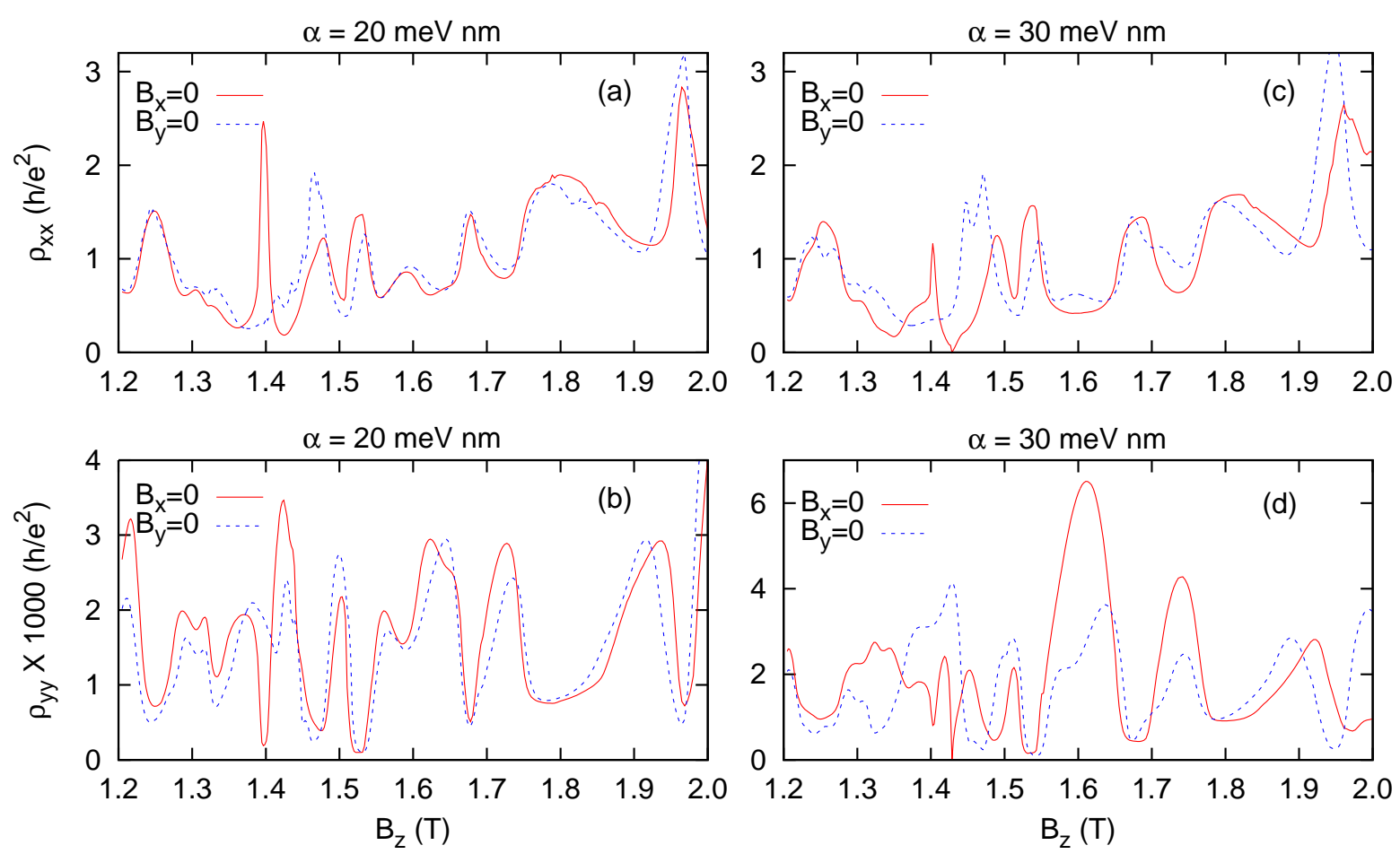

Figure 3. (Color online) Resistivities of the modulated 2DES compared, by tilting the magnetic field along the $y$ or $x$ direction, i. e. when $\varphi=\pi / 2\left(B_{x}=0\right)$ and $\varphi=0\left(B_{y}=0\right)$, respectively. (a) The resistivity along the modulation, i. e. in the $x$ direction. (b) The resistivity in the homogeneous direction $y$, magnified 1000 times. (c) and (d) The resistivities recalculated with the Rashba SOC parameter increased by $50 \%$. In both cases the Dresselhaus SOC strength is $\beta=3 \mathrm{meV} \mathrm{nm}$.

Nevertheless, as expected from the Landau band structure, the message is that both longitudinal resistivities ( $x x$ and $y y$ ) should depend on the orientation of the magnetic field projection in the plane of the 2DES, i. e. on the azimuthal angle $\varphi$.

\section{Magnetization}

The magnetization $\mathbf{M}$ of the modulated 2DES is related to the total free energy of the electrons, $E-T S$, by the thermodynamic relation

$$
d(E-T S)=-\mathbf{M} d \mathbf{B}
$$

where $E$ is the total energy and $S$ the entropy of the 2DES. In the zero-temperature limit we can evaluate the measurable magnetization in the direction perpendicular to the 2DES in a thermodynamic manner [25]:

$$
M_{z}=-\frac{\partial E}{\partial B_{z}} .
$$

The total energy was calculated from the Landau bands as the sum - over all occupied effective single-particle states - of the single-particle energies obtained in the absence 


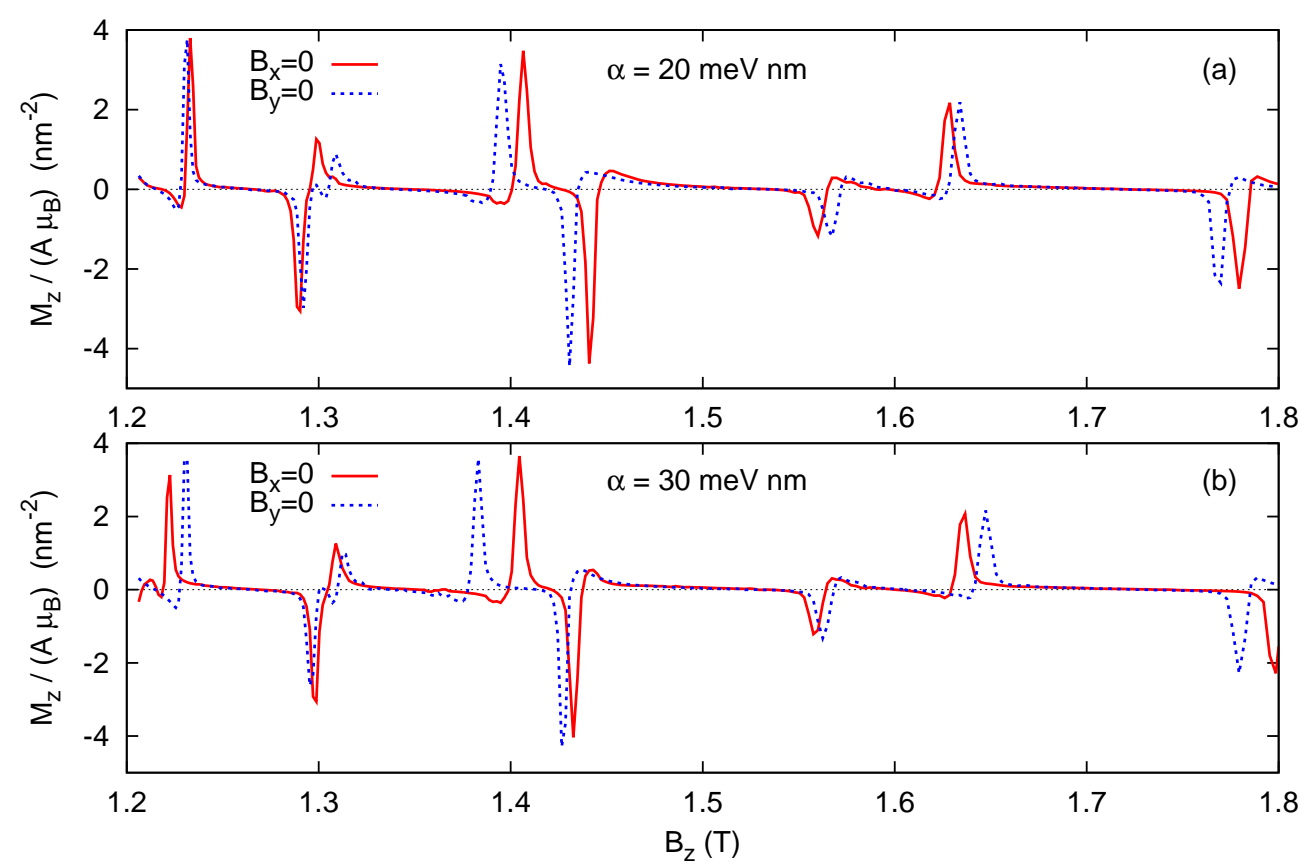

Figure 4. (Color online) (a) The magnetization in the $z$ direction, in units of Bohr magneton $\mu_{B}$, and per surface area $A$ in $\mathrm{nm}^{2}$, vs. the $z$ component of the magnetic field when it is tilted along the $x$ or $y$ directions, i. e. for $\varphi=\pi / 2\left(B_{x}=0\right)$ and for $\varphi=0\left(B_{y}=0\right)$. (b) The magnetization recalculated with the Rashba SOC parameter increased by $50 \%$. In both cases the Dresselhaus SOC strength is $\beta=3 \mathrm{meV} \mathrm{nm}$

of the Coulomb interaction, plus one half of the Coulomb energy in order to exclude the double counting of electrons, like in Eq. 3 of Ref. [26]. Then, according to Eq. 10, the magnetization was obtained with a numerical derivative procedure, on a sufficiently dense grid of magnetic field values.

For a homogeneous 2DES, i. e. in the absence of the periodic modulation, the magnetization has saw-teeth-like de Haas - van Alphen oscillations vs. the $z$ component of the magnetic field, corresponding to the motion of the Fermi energy within a degenerated Landau level, followed by an abrupt jump to the adjacent level. For the modulated system, the magnetization has a positive or a negative peak when the Fermi level touches the bottom or the top of a Landau band, respectively [25].

In Fig. 4 we show the magnetization calculated for the two present situations of interest: when the magnetic field is tilted in the $x$ or in the $y$ direction, i. e. when $\varphi=0$ and $\varphi=\pi / 2$, respectively. The peaks indicate the van Hove singularities of the Landau bands. Comparing to the resistivities, Fig. 3, our calculations predict that the van Hove singularities can be much better resolved in magnetization measurements, even if the Landau bands are partially overlapped, as in Fig. 2, which is the situation in all our calculations.

The oscillations of the magnetization in the modulated 2DES are also quite complex, being determined by the band structure, but indirectly influenced by the Coulomb many- 
body effects. The exchange interaction may lead to larger magnetization fluctuations than expected for noninteracting electrons [25, 26]. Consistently with the dispersion of the Landau bands, due to the presence of the SOC, the magnetization also depends on the azimuthal angle $\varphi$ of the in-plane magnetic field. Like in the transport calculations we considered two values of the Rashba SOC strength for comparison. Since the van Hove singularities of the Landau bands may shift either up or down with increasing $\alpha$, the magnetization peaks may shift either up or down on the magnetic field scale, irrespective of the orientation of the magnetic field.

\section{Conclusions}

We calculated the Landau bands produced in a 2DES by a periodic unidirectional potential in the presence of a tilted magnetic field, with a strong component perpendicular to the 2DES. In the absence of SOC, when the spin contributes only with the Zeeman coupling to the total magnetic field, the Landau bands do not depend on the orientation of the in-plane component of the magnetic field relatively to the direction of the modulation. They depend on the polar angle $\theta$, but not on the azimuthal angle angle $\varphi$, indicated in Fig. 1.

The interplay of the SOC, the unidirectional periodic modulation, and the inplane magnetic field, result in Landau bands whose dispersion explicitly depends on the projections of the magnetic field on axes perpendicular and parallel to the modulation, respectively. Therefore measured observables like electrical resistances or magnetization, in the quantum Hall regime, in a tilted magnetic field, should be sensitive to the rotation of the field, conically, around the normal to the 2DES plane, i. e. to the azimuthal angle angle $\varphi$. Such experimental investigations are reported for the homogeneous 2DES [12], but, to the best of our knowledge, not for the modulated 2DES. We are also not aware of alternative experimental evidence of the asymmetric energy dispersion due to the interplay of SOC and an in-plane magnetic field.

The results that we have shown for the electrical resistance and magnetization are only qualitative. As long as the SOC effects are weak, they become significant only for Landau bands with strong dispersion, produced by a modulation potential considerably larger than the cyclotron energy. Given the complexity of the Landau bands, especially due to the inclusion of the Coulomb effects, it is difficult to make reliable quantitative predictions on the anisotropy of the magnetization or resistances (which are complex observables themselves) in order to relate some features with the SOC strength. The asymmetry under magnetic field orientation could become a diagnostic tool for an estimate of the SOC presence and intensity in the 2DES. Since the van Hove singularities

at the band edges can be well resolved in the magnetization we expect that the SOC effects on the Landau bands could be better observable in magnetization, rather than in magnetotransport measurements.

Finally, the observation of this SOC effect in the magnetoresistance and magnetization experiments proposed in this paper, is likely to depend not only on the SOC 
strength itself, but also on the sample characteristics, such as disorder or electron concentration, details that can only be established in future experimental work.

[1] Zutic I, Fabian J and Das Sarma S 2004 Rev. Mod. Phys. 76323.

[2] Bychkov Yu A and Rashba E I 1984 JETP Lett., 3978.

[3] Dresselhaus G 1955 Phys. Rev. 100580.

[4] Engels G, Lange J, Schäpers T and Luth H 1997 Phys. Rev. B 55 R1958.

[5] Nitta J, Akazaki T, Takayanagi H and Enoki T 1997 Phys. Rev. Lett. 781335.

[6] Winkler R W, Kotthaus J P and Ploog K 1989 Phys. Rev. Lett. 621177.

[7] Zhang C and Gerhardts R R 1990 Phys. Rev. B 4112850.

[8] Wang X F, Vasilpoulos P and Peeters F 2005 Phys. Rev. B 71125301.

[9] Firoz Islam S K 2013 Mod. Phys. Lett. B 271350129.

[10] Dos Santos R W B et al. 2013 Eur. Phys. J. B 86192.

[11] Desrat W et al., 2005 Phys. Rev. B 71153314.

[12] Wilde M A and Grundler D, 2013 New J. Phys. 15115013.

[13] Rupprecht B et al. 2013 Phys. Rev. B 87035307.

[14] Thorgilsson G, Egues J C, Loss D and Erlingsson S I 2012 Phys. Rev. B 85045306.

[15] Fedorov A, Pershin Y V and Piermarocchi C 2005 Phys. Rev. B 7245327.

[16] Pershin Y V, Nesteroff J A and Privman V 2004 Phys. Rev. B 69 121306R.

[17] Bel'kov V V et al. 2005 J. Phys.: Cond. Matt., 173405.

[18] Ganichev S D and Prettl W 2003 J. Phys.:Cond. Matt., 15 R935.

[19] Ogawa N, Bahramy M S, Murakawa H, Kaneko Y and Tokura Y 2013 Phys. Rev. B 88035130.

[20] Nakhmedov E P and Alekperov O 2013 Eur. Phys. J. B 85298.

[21] Giuliani G F and Quinn J J 1985 Phys. Rev. B 316228.

[22] Winkler R 2003 Spin-orbit Coupling Effects in Two-Dimensional Electron and Hole Systems (Berlin: Springer).

[23] Erlingsson S I, Egues J C and Loss D 2010 Phys. Rev. B 82155456.

[24] Manolescu A and Gerhardts R R 1997 Phys. Rev. B 569707.

[25] Gudmundssson V, Erlingsson S I and Manolescu A 2000 Phys. Rev. B 614835.

[26] Meinel I, Grundler D, Heitmann D, Manolescu A and Gudmundsson V 2001 Phys. Rev. B 64 121306(R). 